\title{
Sexualidad juvenil y cambio social: el caso de Portugal
}

José Machado-Pais, D r en Soc.(1)

\section{Machado-Pais J. \\ Sexualidad juvenil y cambio social: \\ el caso de Portugal. \\ Salud Publica Mex 2003;45 supl 1:S26-S33.}

\section{Resumen}

Objetivo. Investigar si, dentro de un marco de profundos cambios sociales, las nuevas generaciones estarán creando nuevos valores sociales. Material y métodos A plicando el método de las generaciones, se utilizaron datos de una encuesta sobre valores y generaciones, hecho en 1996 a 2012 individuos residentes en Portugal continental y representativos de la población portuguesa. Recurriendo al programa SPAD (Estadística para análisis de datos), se procedió a un análisis factorial de correspondencias múltiples en conjugación con un análisis de tipo cluster. Resultados. Constatamos que las principales discontinuidades intergeneracionales se presentan en el área de las actitudes y prácticas sexuales. Comprobamos que los grupos y agregados resultantes del análisis tienen índices de sobrerrepresentación de generaciones específicas. Por ejemplo, los encuestados hedonistas se caracterizan por un elevado índice de sobrerrepresentación de jóvenes, en contraste con los inhibidos o los moralistas. Conclusiones Los jóvenes portugueses aparecen como una generación portadora de valores hedonistas y de carácter experimental en el plano de la vida sexual y amorosa. Sin embargo, se caracterizan al mismo tiempo por su vulnerabilidad a conductas de riesgo.

Palabras clave: cambio social; sexualidad juvenil; gener aciones; Portugal

\section{Machado-Pais J. \\ Youth sexuality and social change: \\ the case of Portugal.}

Salud Publica Mex 2003;45 suppl 1:S26-S33.

\begin{abstract}
A bstract
Objective.To investigate whether the new generations are creating new social values in the current setting of deep so cial change. Material and methods U sing the generations method (Mannheim), a survey was conducted in 1996, among 2012 residents of Portugal, to collect data on values and generations of the Portuguese population. The SPAD statistical analysis program was used to perform a multiple correspondence factorial analysis, in addition to a clustertype analysis. Results The main generational gaps were found in sexual attitudes and practices. Findings showed that groups and aggregates have over representation indices for specific generations, e.g., hedonistic inter viewees are characterized by a high index of overrepresentation of youngsters, in contrast to the moralistic or the inhibited subjects. Conclusions. Por tuguese yo ungsters are depicted as a generation with hedonistic and experimental values regarding love and sex life. However, youngsters are at the same time characterized by both their vulnerability and risk behaviors.
\end{abstract}

Key words: social change; yo uth sexuality; generations; Portugal

(1) Instituto de Ciencias Sociales de la Universidad de Lisboa, Portugal.

Fecha de recibido: 18 de abril de 2001 • Fecha de aprobado: 3 de junio de 2002

Solicitud de sobretiros: Dr. José Machado-Pais. Instituto de Ciencias Sociales, Universidad de Lisboa. Av. Forcas Armadas, edificio I.S.C.T.E. ala sur, 1ro. 1600 Lisboa, Portugal.

Correo electrónico:mac.pais@ mail.telepac.pt 
L a sociedad portuguesa ha vivido, en las últimas décadas, un importante proceso de cambios sociales. Habiendo salido en 1974 de un régimen político de características totalitarias, la sociedad portuguesa se modernizó con la democratización; se desarrolló económicamente con la integración en la Comunidad Europea; se abrió a nuevas corrientes socioculturales destradicionalizándose, especialmente en las áreas de la sexualidad y de la vida amorosa. ${ }^{1}$ ¿Cuál es el protagonismo que las generaciones más jóvenes han tenido en este proceso de transformaciones?

Mannheim, ${ }^{2}$ al desarrollar el método de las generaciones, insistía en que la aceleración de los cambios sociales es un factor importante para la transformación de una "generación potencial" en "generación efectiva". Para Mannheim, las generaciones potenciales, basadas en los ritmos biológicos de la existencia, sólo en circunstancias históricas especiales se transforman en generaciones efectivas, capaces de actuar como fuerzas transformadoras de la sociedad. Mannheim defendía, además, que la aceleración de las transformaciones sociales, con anterioridad y con posterioridad al surgimiento de las generaciones efectivas, no podía dejar de asociarse con nuevas oportunidades de acceso a la cultura por parte de la generación joven. ¿En qué medida las transformaciones que dieron lugar a la Revolución del 25 de abril de 1974, o que ocurrieron con ella, habrán creado un escenario propicio para el protagonismo de los jóvenes portugueses, en un sentido próximo al de generación efectiva? ¿En qué medida la Revolución del 25 de abril habrá tenido marcas específicamente juveniles? Con anterioridad, por intermedio de los movimientos estudiantiles en el reflujo de mayo del 68, y de los movimientos contra la Guerra Colonial; con posterioridad, porque la Revolución de abril liberalizó a la sociedad portuguesa, distendiéndola de sus ataduras políticas más marcantes o represoras. El móvil de las generaciones efectivas -las nuevas oportunidades de acceso a la cultura, según Mannheim-, se dio, de hecho, en la sociedad portuguesa después del 25 de abril, con la escolarización creciente de los jóvenes portugueses.

De esta forma, actitudes y valores que, a primera vista, pueden parecer resultado de un efecto de generación o de edad, en realidad podrán ser también consecuencia de efectos de periodo: transformaciones en las condiciones de escolarización, en el funcionamiento del mercado de trabajo, etcétera. El efecto edad expresa una influencia (de edad) -definida como una posición determinada en el transcurso de la vida- sobre las actitudes, valores, comportamientos. El efecto periodo expresa la influencia de un determinado contexto o periodo ${ }^{3}$ sobre el conjunto de la población que vive bajo el efecto de dicha influencia. El efecto generación se utiliza para destacar rasgos específicos que dan una cierta homogeneidad social y cultural a una generación demográfica. A veces existe una interconexión o una influencia recíproca entre estos varios efectos. Es lo que sucede cuando evaluamos el impacto de acontecimientos de gran magnitud social, como la Guerra Mundial o la Gran Depresión, ${ }^{4}$-en las trayectorias de los individuos. Esta interferencia, de un tiempo social en tiempos individuales asociados con trayectorias de vida, ${ }^{5}$ forma también la base que define a la generación del 25 de abril, o sea, la de los nacidos en la década de la Revolución de los Claveles, entre 1971 y 1980.

En relación con una y otra problemática del estudio, se puede decir que la sociología de la familia ha venido estudiando las relaciones prematrimoniales, la elección del cónyuge, el funcionamiento de las familias, el divorcio, la reconstrucción de las familias. ${ }^{6}$ Pero se sabe poco sobre los aspectos más íntimos de la vida privada, especialmente los relacionados con la vida amorosa y la sexualidad, excepto aquellos que se encuadran bajo una perspectiva que Foucault califica como "represiva" y "utilitarista".

Por este motivo quisimos saber cuáles son las diferentes manifestaciones de la sexualidad que muchas veces se toman como unitaria, y cuáles son las diferentes expresiones del amor en su pluralidad vinculante. ¿Qué representaciones y vivencias tendrían diferentes generaciones sobre tales fenómenos? ¿En qué medida las generaciones más jóvenes serían portadoras de valores diferentes de los característicos de las generaciones de mayor edad?

La problemática inicial se traduce en una tentativa de descubrir diferentes éticas sexuales y amorosas en diferentes generaciones sociales. Las dimensiones analíticas construidas mientras tanto condujeron a algunas hipótesis de investigación: ¿la difusión de los métodos anticonceptivos habría ayudado a la modulación de una nueva sexualidad? ¿Las actitudes en relación con la sexualidad serían de naturaleza más romántica, o más hedonista? ¿Con valores más de confluencia o de conflicto? ¿La "hipótesis de la diversidad" prevalecería sobre la "hipótesis represiva"? ¿Las generaciones jóvenes estarían adoptando una nueva ética sexual y amorosa?

En el abordaje de la problemática enunciada, y en la misma construcción de los indicadores de investigación, se puso de parte la idea que toma la sexualidad como una mera libido natural. Aunque la sexualidad sea vivida corporalmente, los cuerpos y sus prácticas tienen un significado cultural. La sexualidad no es un acto meramente biológico -de los actos más 
repetitivos y banales de la vida cotidiana. Las normatividades de la vida sexual y amorosa se encuentran, de hecho, prescritas y proscritas por diversos espacios, tiempos, modos y ritos.

\section{Material y métodos}

Las encuestas más recientes a la juventud, tanto a escala nacional como internacional, han explorado de forma latente el área de los valores. Pero no ha habido una preocupación fundamental por explicar las variables que llevan a los jóvenes a la adopción de valores diferentes de los de las generaciones de mayor edad, $\mathrm{o}$ sea, valores que explicarían posibles discontinuidades intergeneracionales.

Esta preocupación resultó dominante en una encuesta sobre valores aplicada a las generaciones de la sociedad portuguesa ${ }^{8}$ y cuyos resultados son el material principal de este estudio. La consulta utilizó como universo de estudio a gente de 15 años de edad en adelante, residente en Portugal continental, en localidades con 10 o más habitantes. Del muestreo formaron parte 2012 individuos seleccionados por el método random route, muestreo que fue estratificado por regiones y hábitats (tamaño de las localidades), con selección aleatoria de las localidades, y por cuotas en lo concerniente al sexo, edad y actividad de los encuestados. El trabajo de campo se hizo en 1996.

Las encuestas por cuestionario han sido uno de los instrumentos más utilizados para el estudio de los valores. Sin embargo, las respuestas que se obtienen con ellos son meras opiniones. Pero como los valores traducen representaciones sociales, y teniendo en cuenta el criterio cuantitativo que figura en la definición clásica de representaciones sociales expuesta por Moscovici, ${ }^{9}$ podemos considerar las opiniones ampliamente compartidas como susceptibles de apuntar valores sociales básicos.

Por otra parte, el instrumento metodológico utilizado - una consulta basada en un cuestionario- nos obliga a tomar el concepto de generación en uno de sus sentidos más simples, haciéndolo recubrir un conjunto de clases por edades: ${ }^{10}$ se trata de una definición demográfica de generación que puede contraponerse a otras definiciones de ésta. Al discutir los criterios que nos permiten definir una representación social, Moscovici sugiere tres: el criterio cuantitativo, que permite ocuparse de la extensión de una representación social en una determinada colectividad; el criterio de la producción, según el cual la representación social puede considerarse como expresión de un determinado colectivo social y el criterio funcional, que permite evaluar la contribución propia de la representación social en un determinado proceso de socialización, es decir, en el proceso de formación y orientación de las conductas sociales. Sólo podemos discutir, indirectamente, la problemática de las generaciones en un sentido sociológicamente más refinado, como hizo Mannheim al proponer el concepto de "generaciones efectivas", en el marco de un proceso dinámico de desestabilización o cambio social.

Pasando ahora a la operatividad del método de las generaciones, un análisis factorial de correspondencias múltiples (tipo cluster) que contempló las variables sociográficas de la encuesta (grupo etario, género, estado civil, ocupación y nivel de estudios), y todos los indicadores relacionados con la sexualidad y la vida amorosa de los portugueses, dio origen a un dendograma con 12 principales clases de distribución. El programa SPAD (Estadística para análisis de datos) complementa un análisis factorial de correspondencias múltiples con un análisis de clasificación hierárquica (cluster). Los individuos encuestados y sus modalidades de respuesta (todas ellas tomadas como variables de análisis) fueron agrupados en clases, de acuerdo con los resultados del análisis. Cada clase agrega, por consiguiente, las modalidades de respuesta sobrerrepresentadas según el valor-teste (se trata de un teste estadístico semejante al ji-cuadrada). Esta sobrerrepresentación ocurre cuando las modalidades de respuesta tienen frecuencia más elevada que las esperadas bajo la hipótesis de independencia. De dicha distribución en 12 clases, resultante de la aplicación del programa SPAD, adquieren especial relevancia tres agregados que propondríamos como definidores, a efectos de análisis, de los siguientes tipos sociales: los inhibidos, los moralistas y los hedonistas. Los tres agregados son resultado del análisis de la clasificación jerárquica (análisis de cluster) que se basa, en un análisis factorial de correspondencias múltiples. Los "tipos" son meras designaciones nominales de los agregados. (Figura 1).

El análisis multivariable demostró que los grupos y agregados resultantes del análisis tienen índices de sobrerrepresentación de generaciones específicas: la generación del 25 de abril, que comprende encuestados nacidos en la década de la Revolución, entre 1971 y 1980; la generación de la Guerra Mundial, que comprende encuestados nacidos antes de 1941; y, finalmente, una generación de transición, con encuestados nacidos entre 1941 y 1971 (cuadro I).

\section{Resultados}

Veamos entonces cuáles son las características de los tipos sociales encontrados. Los inhibidos (tipo resul- 


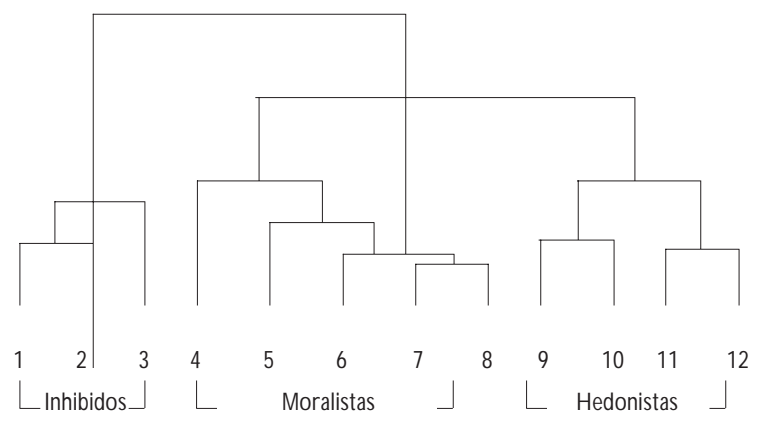

Figura 1. Dendograma de vida sexual y amorosa. Portugal continental, 1996

tante de la agregación de las clases 1, 2 y 3) se definen principalmente por su renuncia sistemática a responder las preguntas de la encuesta relacionadas con la temática de la sexualidad: representaciones sobre la sexualidad (ex: amor vs. placer), homosexualidad, adulterio, relaciones sexuales extraconyugales; aspec- tos considerados más importantes en la sexualidad (placer sexual, amor, erotismo, orgasmo, envolvimiento romántico, etcétera); edad de iniciación sexual; cantidad de personas con quien ha tenido relaciones sexuales; sitios donde ocurrieron las relaciones sexuales; uso de métodos contraceptivos; actitudes ante la pornografía; etcétera. Aunque en términos generales los inhibidos tienden a no aceptar que un hombre o una mujer casados tengan relaciones sexuales extramatrimoniales (explícitamente los del grupo 1, mientras que los del grupo 2 no se manifiestan), se dividen en relación con otros indicadores de la siguiente forma: el grupo 1 tiende a condenar la homosexualidad masculina y femenina, la prostitución, y la masturbación masculina, no estando de acuerdo en que la pornografía pueda contribuir para mejorar la vida sexual; el grupo 3, como contrapartida, se muestra tolerante con la homosexualidad y con la masturbación masculina y femenina; acepta que una mujer pueda pagar para tener relaciones sexuales; y está de acuerdo en que la pornografía pueda mejorar la vida sexual.

La postura más liberal del grupo 3 se debe al hecho de estar formado principalmente por jóvenes (de

Cuadro I

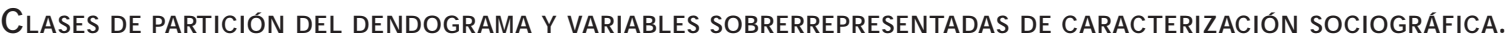
Portugal continental, 1996

\begin{tabular}{|c|c|c|c|c|c|}
\hline Clases & Grupo de edad & Género & Estado civil & Ocupación & Nivel de estudios \\
\hline 1 & $\begin{array}{l}40-49 \\
55-59 \\
65-74\end{array}$ & & $\begin{array}{l}\text { Casado/iglesia } \\
\text { Casado/civil }\end{array}$ & Ama de casa & Primario analfabeto \\
\hline 2 & & $\mathrm{~F}$ & Casado/iglesia & & \\
\hline 3 & $15-29$ & M & Soltero & Estudiante desempleado & Secundario universitario \\
\hline 4 & & $\mathrm{~F}$ & & & Secundario \\
\hline 5 & & $\mathrm{~F}$ & Soltera & Estudiante ama de casa & Secundario \\
\hline 6 & & $\mathrm{~F}$ & Casado/civil & A ma de casa jubilado & Primario analfabeto \\
\hline 7 & $\begin{array}{l}50-54 \\
65-74\end{array}$ & $\mathrm{~F}$ & $\begin{array}{c}\text { Viuda } \\
\text { Casado/civil } \\
\text { Casado/iglesia }\end{array}$ & $\begin{array}{l}\text { Jubilado } \\
\text { Ama de casa }\end{array}$ & Primario \\
\hline 8 & $65-74$ & $\mathrm{~F}$ & Viuda & Ama de casa & A nalfabeto primario \\
\hline 9 & $25-29$ & M & & Desempleado & Secundario \\
\hline 10 & $\begin{array}{l}35-39 \\
50-54\end{array}$ & M & & & Primario \\
\hline 11 & $20-29$ & & Soltero cohabitante & Estudiante & Secundario \\
\hline 12 & $20-29$ & M & Soltero & & $\begin{array}{c}\text { Secundario } \\
\text { Universitario medio }\end{array}$ \\
\hline
\end{tabular}


los 15 a los 29 años de edad), todos ellos solteros, con niveles de estudios que llegan a alcanzar la enseñanza superior. El rechazo a responder las preguntas sobre sexualidad, si no se comprende muy bien en el caso del grupo 3, se adivina con facilidad en el caso de los grupos 1 y 2: en el primero nos encontramos ante encuestados que se distinguen por ser de edad avanzada (algunos jubilados), casados (en algunos casos, empleadas de hogar) y analfabetos o sólo con el nivel de instrucción primaria (en 52\% de los casos); en el segundo grupo tenemos la figura tradicional de la mujer ( $80 \%$ en el grupo considerado), que (en 55\% de los casos) es ama de casa; en este último grupo están también sobrerrepresentados los casados por la iglesia católica. Estos dos grupos tienen, pues, una mayor sobrerrepresentación entre los encuestados de la generación de la Guerra Mundial, nacidos antes de 1941.

Los moralistas (tipo resultante de la agregación de los grupos 4, 5, 6, 7 y 8) tienen también una sobrerrepresentación de la generación de la Guerra Mundial (principalmente los grupos 7 y 8). Otra característica esencial y transversal a todos estos grupos es la sobrerrepresentación femenina. Los moralistas se caracterizan por adoptar un patrón de actuación con base sólidamente moralista: de una manera general, tienden a aceptar relaciones sexuales sólo entre hombres y mujeres después del matrimonio; reprueban el adulterio; en las relaciones sexuales valoran sobre todo el amor entre los cónyuges, el nacimiento de los hijos y el vínculo romántico; imponen condiciones necesarias para que dos personas puedan mantener relaciones sexuales (que estén casadas, que cohabiten, que mantengan un noviazgo o, por lo menos, que se amen); la iniciación sexual tiende a darse (concretamente en los grupos 6, 7 y 8) a los 21 años de edad o más, o con el(la) novio(a) o con el(la) esposo(a), o sea, en los "pórticos" del matrimonio; no tuvieron relaciones sexuales con nadie más aparte de la pareja con la que se iniciaron. En el caso particular de los consultados de los grupos 7 y 8 -caracterizados por estar formados por los de edad más avanzada- los rasgos de marca son: no se recurre a preservativos, sobre todo por no haber necesidad (nótese que predominan las viudas en estos dos grupos); en relación con el SIDA no saben lo que eso representa; no aceptan que un hombre casado (mucho menos una mujer casada) tenga relaciones extramatrimoniales; no toleran (o rehúsan responder a preguntas sobre comportamientos sexuales que se salen del patrón moral de lo que consideran ser una sexualidad "normal", es decir, no aceptan la homosexualidad, la masturbación ni la prostitución); finalmente, en relación con la pornografía, la rechazan basándose en to- dos los argumentos posibles (crea vicios, es inmoral, etcétera).

La sociografía de los moralistas apunta hacia un universo típico: el de las amas de casa. Otro rasgo que los caracteriza es el bajo nivel de estudios. También se encuentran excluidos del mercado de trabajo (debido al gran número de empleadas de hogar y jubilados). En los grupos de tendencia más moralista (6 y 7) es relevante el peso de viudas y de residentes en una región pobre del país (Alentejo).

Finalmente, tenemos los hedonistas. Forman parte de este tipo los grupos 9, 10, 11 y 12. Se caracterizan por un elevado índice de sobrerrepresentación de jóvenes. Se trata, pues, del cluster que acoge a la generación de abril, es decir, de los encuestados nacidos entre 1971 y 1980, con edades comprendidas entre los $15 \mathrm{y}$ los 34 años en el momento en que fueron consultados. La generación de transición, en el caso de los hombres, es predominante en el grupo 10 de los hedonistas.

En cuanto al sexo, los hedonistas tienden a valorar el placer; de una manera general aceptan todo tipo de relaciones sexuales; defienden abiertamente las relaciones sexuales antes del matrimonio y se muestran tolerantes en relación con el adulterio. Un rasgo distintivo de los hedonistas es la valoración del orgasmo. Pero en general tienden también a valorar -excepto la procreación- todas las funciones, manifestaciones, goces o temores asociados con la sexualidad: placer, amor entre la pareja, dominio de las técnicas sexuales, erotismo, satisfacción sexual de la pareja, satisfacción de una necesidad biológica, relación romántica, miedo de adquirir SIDA y de provocar embarazos no deseados.

Entre los hedonistas adquiere relevancia la experiencia sexual y se pone de manifiesto una iniciación sexual precoz que tiende a darse con amigos(as) y novios(as) y que se multiplica en varias relaciones sexuales. Otro de sus rasgos característicos es la diversidad de lugares en los que ya mantuvieron relaciones sexuales. Este rasgo es característico de grupos que están en la vanguardia de la llamada sociedad "posmoderna" basada, como sugiere William Simon, en una intensa pluralización, individualización y multiplicidad de elecciones. ${ }^{11}$ Por necesidad (recurso) o por deseo (opción) los hedonistas tuvieron ya relaciones sexuales en coches, en la playa, en el campo, en casa (propia, de los padres, de la pareja, o de algún conocido), hoteles, jardines, escuelas, calles, barcos, escaleras de edificios y lugares de prostitución.

Aparentemente resulta extraño que entre los hedonistas de los grupos 9 y 10 se rechace el uso de pre- 
servativos. Sin embargo, parece aquí haber también una lógica de placer sexual que determina tal rechazo. Por lo menos algunos de los consultados apuntan como razón del rechazo al preservativo la falta de placer (propio o de la pareja). Los hedonistas son también los que mejor aceptan las relaciones extramatrimoniales, la prostitución y la homosexualidad (en este caso, con excepción del grupo 10). La aceptación de la masturbación -masculina o femenina- es también un rasgo característico de éstos, en porcentajes que oscilan entre $60 \%$ y más de $80 \%$ de aceptación. Y se comprende el porqué. Las interdicciones masturbatorias o los correspondientes discursos inhibidores son metáfora de regímenes disciplinarios de la sexualidad. Para muchos sexólogos, la masturbación es sólo una forma de experimentación de la sexualidad, mediante pensamientos, deseos, gratificaciones. ${ }^{12}$ Como tal, tiene cabida en una ética hedonista. Por las mismas razones, los hedonistas se muestran favorables a todos los predicados que valoran la pornografía (mejora la vida sexual, proporciona diversión, etcétera).

Contrastando con los moralistas (tipo marcadamente femenino), a los hedonistas les corresponde un perfil de masculinidad. Pero el distintivo más destacado apunta hacia el hecho de que estos últimos son más instruidos y más jóvenes. La excepción se encuentra entre los consultados del grupo 10, que denominaríamos hedonistas machistas. En efecto, se distinguen por un nivel cultural muy bajo (en este grupo destacan los que, en cerca de $60 \%$ de los casos, tienen sólo instrucción primaria), y son de una edad con mayor frecuencia comprendida entre los 35 y los 54 años; de condición social diversificada, el hedonista machista puede ser tanto un propietario como un obrero cualificado, un comerciante, un agricultor o un pescador. $\mathrm{Al}$ unir un declarado dominio de las técnicas sexuales a la valoración del orgasmo, los hedonistas machistas consideran que para que una pareja tenga relaciones sexuales es suficiente con que se conozcan y se deseen o busquen solamente pasar un buen rato -como si basaran toda la vivencia sexual en la arqueología del deseo. Como corolario, brillan por el número de compañeras con las que (dicen) se habían relacionado: más de 20 en algunos casos, y otros perdieron la cuenta. La iniciación sexual del hedonista machista se dio de los 10 a los 15 años de edad implicando, de forma más recurrente, a una amiga ( $27 \%$ de los casos) o desconocida ( $54 \%$ de los casos), eventualmente una trabajadora del sexo comercial. Es además entre ellos donde más se da el frecuentar sitios de prostitución (visitados por $45 \%$ de los consultados de este grupo) y, naturalmente, aceptan que un hombre (¡no una mujer!) pague para tener relaciones sexuales. Sin embargo, no usan preservativo porque, alegan, les resta placer. De entre los grupos agregados en la tipología de hedonista, los machistas (grupo 10) son los únicos que no aceptan explícitamente la homosexualidad, especialmente la homosexualidad masculina, rechazada por más de $90 \%$ de los miembros de este grupo. O sea, su machismo impone límites al hedonismo libertario.

Los grupos 11 y 12 definen un hedonismo sustantivamente más cultivado. El grupo 12 se distingue del grupo 11 por tener una sobrerrepresentación de encuestados de sexo masculino. Por consecuencia, en el grupo 11 hay una sobrerrepresentación de encuestados que afirman que la persona con quien se relacionaran sexualmente por primera vez era novio (valor teste: 14.3) mientras que en el grupo 12 las primeras relaciones sexuales suelen ocurrir con amigos (valor teste: 12.1). Este subagregado tiene una presencia considerable de jóvenes, con un nivel de instrucción que llega a alcanzar la enseñanza media o superior. Algunos tuvieron su iniciación sexual en la escuela. Son los únicos que subrayan de forma más clara que el SIDA es una enfermedad como cualquier otra. Les parece intolerable que un hombre tenga que pagar para tener relaciones sexuales -no precisamente por razones morales (como sucederá con los moralistas), sino por razones simbólicas (les parecerá, probablemente, un desprestigio el recurso a la prostitución).

De los jóvenes de los 15 a los 24 años de edad, sólo $20 \%$ respondió que nunca había tenido relaciones sexuales. Tomando el universo de los consultados que confesaron que ya habían tenido relaciones sexuales $(n=1715)$, tratamos de averiguar a qué edad se había dado la iniciación sexual. Como es sabido, las primeras experiencias sexuales pueden ser significativas en el futuro de la vida individual de las personas y, en esa medida, son un signo revelador de comportamientos sexuales futuros. La primera relación sexual tiene una carga simbólica equivalente a la de un "rito de tránsito" -no de un tránsito meramente biológico, sino social- o no se estaría tratando de un acontecimiento que está inscrito en un contexto generacional y social. ${ }^{13}$

Los datos de la encuesta realizada demuestran que la edad de la primera relación bajó considerablemente en términos generacionales. De ahí que, en la generación del 25 de abril (nacidos entre 1971 y 1980), $67 \%$ se inició sexualmente antes de los 18 años de edad; como contrapartida, en la generación de la Guerra Mundial (que comprende los nacidos antes de 1941), sólo $21 \%$ se inició antes de los 18 años. O sea, entre las dos generaciones consideradas existe una diferencia porcentual de $46 \%$ en lo referente a la edad de la iniciación sexual.

También la pertenencia generacional parece condicionar la elección del tipo de persona con la que se tiene relaciones sexuales por primera vez. Entre los 
consultados con más de 45 años de edad -y porque entre ellos hay una sobrerrepresentación de casados- el(la) esposo(a) aparece como el protagonista que más se menciona. Ya entre los más jóvenes (menos de 25 años de edad), la iniciación sexual tuvo lugar, predominantemente, con el(la) novio(a) (52\%) o con un(a) amigo(a) (27\%). Finalmente, los datos de la encuesta demuestran una acentuada heterogamia en la generación más joven (15 a 24 años de edad). En efecto, $49 \%$ de los jóvenes consultados ya tuvo relaciones sexuales con más de un(a) compañero(a) y $21 \%$ con más de cinco.

\section{Discusión}

Los datos de la encuesta sobre los valores y generaciones sugieren que la sociedad portuguesa atraviesa por importantes discontinuidades intergeneracionales cuando se discuten las representaciones sobre la sexualidad y la vida amorosa.

En una sociedad gobernada por una moral rígida, como lo fue la sociedad portuguesa hasta los años 70, deberían aún surgir manifestaciones residuales de cierta intolerancia en cuanto a las relaciones prematrimoniales. La generación de la Guerra Mundial transmite esta mentalidad prohibitoria. No obstante, la generación que llegó a la pubertad a lo largo de los años 80 (generación de abril) es tolerante ante la posibilidad de que las relaciones sexuales tengan lugar antes del matrimonio. Aquí tenemos realmente "efectos de periodo" (resultado de la Revolución) determinando las actitudes de los jóvenes nacidos en la década de la Revolución.

Diversos factores han contribuido para recurrir más a las relaciones prematrimoniales, como lo prueba la teoría de los "medios, motivos y oportunidades". Basándose en algunas teorías criminológicas y comparando los factores que llevan al crimen con aquellos que hacen viables las relaciones prematrimoniales, Robert Walsh ${ }^{14}$ llega a la conclusión de que, en ambos casos, los medios, los motivos y la ocasión..., hacen al "ladrón" (aún persiste la representación social de que la virginidad es robada). En el plano de la sexualidad, Walsh mantiene que los medios se manifiestan, entre los jóvenes, a edades más precoces (una madurez biológica más rápida); los motivos son aumentados por "grandes focos" publicitarios (tv, cine, videos, música); en cuanto a las oportunidades, no van faltando: o por la creciente incorporación de la mujer al sistema educativo y al mercado de trabajo, o porque los hijos permanecen en casa "menos cohibidos", o porque la divulgación de los métodos anticonceptivos aleja el temor a embarazos no deseados.
Los datos de la encuesta muestran que la iniciación sexual se ha anticipado bastante a la inserción conyugal. La generación de abril (la de los jóvenes) llega al matrimonio con una ya amplia experiencia sexual, lo que no sucedía, en tan grande extensión, con la generación de la Guerra Mundial, especialmente el componente femenino. En el pasado había una transición más brusca entre un pequeño periodo de (posible) iniciación sexual y el matrimonio. La iniciación sexual entre los jóvenes se basaba en encuentros furtivos y clandestinos. Actualmente, la sexualidad es experimentada por los jóvenes normalmente antes del matrimonio.

La iniciación sexual entre las jóvenes generaciones, fuera del marco matrimonial, y las propias experiencias de cohabitación pueden ser formas de iniciación y experimentación de una vivencia matrimonial. La cohabitación misma parece no implicar sólo a "cohabitantes ideológicos", ${ }^{15}$ sino a compañeros que tenderán a optimizar el matrimonio, ritualizándolo informalmente o experimentalmente mediante la cohabitación.

En suma, los jóvenes parecen ser portadores de una nueva ética sexual, bastante más desinhibida o tolerante que aquella que caracteriza a las generaciones que les preceden. En este sentido, puede afirmarse que la generación de abril presenta marcas de una generación efectiva, para utilizar el concepto de Mannheim. Se diría que -aunque se trata de una hipótesis que merece una mayor profundización en investigaciones posteriores- mientras que las generaciones de más edad están orientadas por valores que radican en un ideario de colectivismo social, las generaciones más jóvenes abrazan valores más fluctuantes que se basan en un individualismo social. ${ }^{16}$ En el primer caso parece darse una subordinación de las aspiraciones individuales a causas colectivas: los derechos sociales, las identidades comunitarias, las dependencias emocionales. En el segundo caso parece darse una subordinación de las causas colectivas a aspiraciones individuales: realización personal, derechos privados, iniciativas individuales.

Entre las generaciones de mayor edad, el ideario de colectivismo social da cobertura a una ética sexual conservadora, defensora del matrimonio institucional, de la estructura familiar tradicional, de las uniones duraderas, de un puritanismo sexual. Entre las generaciones más jóvenes -y porque la "modernidad" se asocia con una "cultura de la separación", ${ }^{17}$ - el ideario del individualismo social estaría más próximo de una ética sexual experimentalista y fragmentada donde hay cabida para relaciones fugaces y románticas; experiencias prematrimoniales y cohabitacionales, iniciaciones sexuales precoces y relaciones heterógamas; 
siendo, apreciándose finalmente una relativa tolerancia hacia diversas formas de sexualidad social o ideológicamente consideradas más "periféricas".

A un modelo de iniciación sexual tradicional asociada con el matrimonio, le sigue, pues, un modelo de "precocidad moderna", ${ }^{18,19}$ en la que la iniciación sexual es facilitada ${ }^{1}$ por la divulgación de métodos anticonceptivos y por una mayor libertad sexual.

Como ya se ha afirmado, los datos de la encuesta demuestran una acentuada heterogamia en la generación más joven (15 a 24 años de edad). La cuestión que se plantea es la de saber si los jóvenes se encuentran preparados -en la escuela o en la familia- para evitar embarazos no deseados o enfermedades de transmisión sexual. Es muy probable que un número considerable de padres y de madres persista en encarar la sexualidad de sus hijos -y principalmente de las hijas- como si su iniciación sólo tuviera lugar con el matrimonio. Corroborando esta hipótesis, tenemos que en Portugal se registra uno de los mayores porcentajes de madres solteras de la Comunidad Europea.

O sea, los patrones tradicionales de la moral sexual -que orientan las actitudes de los padres de los jóvenes en relación con la sexualidad- entran en abierto conflicto con la sexualidad más hedonista que los jóvenes viven, resultando de ello los embarazos inesperados. ${ }^{20}$

De todo ello podemos concluir que la mayor libertad sexual vivida por parte de los jóvenes aparece asociada con algunas conductas de riesgo. En efecto, en una encuesta a los jóvenes de Loures, uno de los municipios más populosos de los alrededores de Lisboa, constatamos que 37\% de los consultados ya había recurrido al "coitus interruptus" ${ }^{21}$ Este dato puede también ser indicio de que algunos jóvenes estén desarrollando una sexualidad dominada por la frustración, por la suspensión del placer, con todas sus inevitables y nefastas cargas de inhibición y de temor.

\section{Referencias}

1. Machado-Pais J. Culturas Juvenis. Lisboa: Imprensa N acional Casa da Moeda, 1991.

2. Mannheim K. Le Problème des Générations. París: Ed. N athan, 1990:1928.

3. Funes-Rivas MJ. Ciclo vital y acción colectiva. Rev Int Sociol 1995;12:

29-54.

4. Elder GH. Children of the Great Depression. Chicago: University of Chicago Press, 1974.

5. Mannheim K. Essays in the Sociology of Knowledge. Londres: Routledge and Kegan Paul, 1952.

6. De Singly F, dir. La Famille. L'État des Savo irs. París: La D écouverte, 1991.

7. Foucault M. Historia de la Sexualidad. Madrid:Siglo Veintiuno de España Editores, 1980.

8. Machado-Pais J, coord. Gerações e Valores na Sociedade Portuguesa Contemporânea. Lisboa: Secretaria de Estado da Juventude, 1998.

9. Moscovici S. La Psychanalyse, son Image et son Publique. París: Presses Universitaires de France, 1961

10.Attias-D onfut C. Générations et Ages de la Vie. París: PUF, 1991.

11. Simon W. Postmodern Sexualities. Londres: Routledge, 1996.

12. Kaplan JL.Adolescence.The Farewell to Chlidhood. N ueva York:Jason Aronson, 1988.

13. Bozon M. Léntrée dans la sexualité adulte: le premier rapport et ses suites. Population, 1993; 5: 1317-1352.

14.W alsh RH . Premarital sex among teenagers and young adults. En: Mckinney K, Specher S, ed. Human Sexuality. The Societal and Interpersonal Context. N ueva Jersey (N J):A blex Publishing Corporation, 1994. 162-186. 15. Roussel L, Bourguiznon 0. Générations Nouvelles et Mariage Traditionnel. Enquête de Jeunes de 18-30 ans. París: IN ED, 1978.

16. Dion KK, Dion KL. Individualistic and collectivistic perspectives on gender and the cultural context of love and intimacy. J Soc Issues 1993; (49)3:53-69.

17. Bellah R. Hábitos del corazón. Madrid:Alianza, 1989:215.

18. Bozon M. Les femmes et l'écart d'age entre conjoints. Une domination consentie: I-Types d'union et attentes en matière d'ecart d'age. Population 1990;2:327-360.

19. Bozon M. Les femmes et l' écart d'age entre conjoints. Une domination consentie:II-Modes d'entrée dans la vie adulte et représentations du conjoint. Population, 1990;3:565-602.

20. Machado-Pais J, coord. Traços e Riscos de Vida. Porto:A mbar, 1999.

21. Machado-Pais J, Ferreira de Almeida J,Torres A, Machado FL,AntunesFerreira P, Sedas-N unes J. Jovens de Hoje e de Aqui. Loures: Câmara Municipal de Loures, 1996. 